\title{
El control de la Administración en tiempos de pandemia
}

\author{
Administration control in pandemic times
}

José Carlos Moslares ${ }^{1}$

\section{Resumen}

El objeto del presente ensayo es realizar un análisis del control de la Administración en el marco de la pandemia de la COVID-19. La emergencia como podremos observar presupone per se la necesidad de brindar respuestas inmediatas pero que de ningún modo pueden validar el incumplimiento de la normativa vigente. Lo que esta sí habilita es el ejercicio de facultades excepcionales para remediar y poner fin a tal situación. Es en esa utilización de remedios excepcionales en los que necesariamente van a encontrarse afectados por un lado derechos y garantías de raigambre constitucional y, por otro, la plena vigencia del sistema republicano de gobierno que presupone la división de las funciones de poder y el control recíproco de estas. La emergencia y las medidas adoptadas para afrontarla necesariamente deben realizarse en el marco del Estado convencional de derecho, y la presencia e intervención de los organismos de control se torna, a nuestro entender, imprescindible. También se realiza un análisis de la función de los organismos de control, los que en la situación de emergencia deben asumir su rol coadyuvante en pos de la actuación de la Administración en el marco de la legalidad que debe guiar siempre su accionar.

Palabras clave: emergencia - control público - validez constitucional

\begin{abstract}
The purpose of this essay is to carry out an analysis of the Administration's control in the context of the COVID-19 pandemic. The emergency, as we can see, presupposes per se the need to provide immediate responses, but that in no way can validate the non-compliance with regulations in force. What it does enable is the exercise of exceptional powers to remedy and put an end to such situation. It is in this use of exceptional remedies that, on one hand, constitutional rights and guarantees will necessarily be affected and, on the other hand, the full validity of the republican system of government that presupposes the division of the functions of power and their reciprocal control. The emergency and the measures adopted to face it must necessarily be carried out within the framework of the conventional rule of law, and the presence and intervention of the control agencies becomes, in our opinion, essential. An analysis is also carried out of the function of the control agencies, which in the emergency situation must assume their role as coadjuvants in pursuit of the Administration's action within the framework of the legality that must always guide their actions.
\end{abstract}

Keywords: Emergency - public control constitutional validity

\section{Derecho/ ensayo}

Citar: Moslares, J. C. El control de la Administración en tiempos de pandemia. Omnia. Derecho y sociedad, 4 (4), pp. 79-90.

\footnotetext{
Abogado. Especialista en Derecho Administrativo Económico. Docente en las cátedras Derecho Constitucional y Derechos Humanos y Garantías, de la Facultad de Ciencias Económicas y Jurídicas, y en la asignatura Legislación, de la carrera de Ingeniería, ambas de la Universidad Nacional de La Pampa. Autor de distintos artículos referidos esencialmente a la temática del derecho constitucional y del control público. Vocal del Tribunal de Cuentas de La Provincia de La Pampa. jmosla@hotmail.com
} 


\section{INTRODUCCIÓN}

La pandemia de la COVID-19 afectó, afecta y afectará nuestras vidas y también la vida de nuestras instituciones.

El análisis que aquí proponemos se centrará en la observación de la práctica institucional puntualmente referida al funcionamiento de los distintos órganos de control sobre los poderes del Estado y la interrelación de estos en el marco de la pandemia.

Por ello, el objeto del presente trabajo es realizar un somero relevamiento de la legislación que da marco a la emergencia y de cuáles son las condiciones de su validez constitucional (y convencional).

La importancia de este análisis se centra en la gestión de los distintos poderes que, aún en situaciones de pandemia, no debe exceder de modo alguno las competencias legal y constitucionalmente asignadas; porque ello implicaría vulnerar el principio de legalidad rector, en tanto las competencias de los distintos órganos de gobierno deben estar establecidas por ley.

Se hará un especial énfasis en la intervención de los distintos órganos de control que de algún modo operan como un reaseguro del funcionamiento del mismo sistema republicano de gobierno.

Su especial relevancia queda de manifiesto en un contexto de emergencia, donde el poder administrador encuentra habilitadas competencias excepcionales para su remediación y atención, lo que exige la presencia de un control oportuno.

\section{CONTROL PÚBLICO}

Primeramente, es importante conceptualizar qué comprende el control y de qué modo se ejerce, para así poder demostrar su relevancia.
El control es uno de las funciones más importantes en la vida institucional de un país, desde nuestro punto de vista, para la defensa de los intereses de la sociedad y el correcto (y legal) funcionamiento de poder estatal.

El control en sí mismo tiene fundamento constitucional y no caben dudas de que tiene íntima vinculación con la forma de gobierno representativa y republicana.

También reconoce raigambre constitucional en las cláusulas constitucionales denominadas de progreso y bienestar, en aquello que la Constitución Nacional establece como la necesidad de promover la prosperidad general (artículo 75, incisos 18 y 19).

La idea de control de la Administración Pública está vinculada con la idea del sistema republicano, con la división de poderes, pero también con el principio de publicidad de los actos de gobierno.

Publicidad y transparencia van de la mano; cuanta más publicidad, más y mejor control. Estas funciones se ejercen entre los distintos poderes de manera recíproca bajo aplicación de la teoría conocida como checks and balances (pesos y contrapesos), donde lo que se busca es que ningún poder prevalezca por sobre los demás, ni aun en épocas excepcionales como una pandemia.

Esta función de pesos y contrapesos opera como una función preventiva en primera instancia, y como correctora si ocurre algún desvío del poder o un avasallamiento de los demás poderes.

Quien mayoritariamente tiende a incurrir en estos abusos o excesos es el Poder Ejecutivo, justificándose mayormente en la necesidad de celeridad en la respuesta. Para prevenir excesos entendemos que la función de control requiere el funcionamiento pleno de los distintos poderes y las distintas instancias de control. 
Así, la rendición de cuentas constituye una herramienta imprescindible en este análisis, ya que la habilitación, vía delegación, de competencias que realizó el Poder Legislativo a favor del Poder Ejecutivo de ningún modo puede significar la omisión de este deber.

Es dable recordar que la función de control se basa en el principio jurídico según el cual quien administra un patrimonio ajeno estará obligado a rendir cuentas de sus actos y del resultado de su gestión al titular de los bienes administrados. Y la función de control no se relaja o exime en una situación de emergencia. Por ello, en este trabajo vamos a bregar por el funcionamiento de las instituciones que no solo aseguren una respuesta inmediata, sino la intervención oportuna de todas las instancias de control que correspondan en resguardo de la legalidad que debe regir toda actuación de la Administración.

\section{EMERGENCIA}

No existe en la Constitución Nacional una definición de emergencia que pueda servir para conceptualizarla para determinar su alcance. ¿Por qué es importante definirla? Porque allí encontraremos una manera de ejercer un control sobre los actos que se realicen dentro de su marco.

En nuestro país, Argentina, solemos decir que vivimos en una «permanente situación de emergencia» — mayormente económica-, la cual, justificada en múltiples razones, ha llevado a la desnaturalización de dicho instituto. En conclusión, lo extraordinario pareciera haberse tornado «normal».

No obstante, la situación de excepcionalidad que nos encontramos transitando escapa a la conclusión expuesta anteriormente; la pandemia declarada por la Organización Mundial de la Salud justifica la emergencia dictada y el uso pleno de las facultades extraordinarias que tal situación amerita.

Buscamos, entonces, desde una mirada constitucional de la emergencia declarada, reflexionar sobre cómo conviven los derechos y garantías constitucionales y convencionales, y de qué manera pueden desarrollarse las tareas de control sin que ello implique entorpecer el funcionamiento de la Administración en momentos en que se exige mayor celeridad en su respuesta; celeridad que de ningún modo puede implicar apartarse o desconocer la normativa vigente.

Al momento de definir el concepto de emergencia, lo podemos hacer referenciando una situación no prevista que, atento a su naturaleza extraordinaria, amerita el uso de facultades excepcionales para su remediación.

La Corte Suprema de Justicia de la Nación la ha definido en el fallo «Peralta, Luis Arcenio y otro c/ Estado Nacional (Mrio. de Economía BCRA) s/ amparo»²:

... el concepto de emergencia abarca un hecho cuyo ámbito de aplicación temporal difiere según circunstancias modales de épocas y sitios. Se trata de una situación extraordinaria, que gravita sobre el orden económico social, con su carga de perturbación acumulada, en variables de escasez, pobreza, penuria o indigencia, originada en un estado de necesidad al que hay que ponerle fin. La etiología de esta situación, sus raíces profundas y elementales, y en particular sus consecuencias sobre el Estado y la sociedad, al influir sobre la subsistencia misma de la organización jurídica y política, o el normal desenvolvimiento de sus funciones, autoriza

2 Fallos: 313:1513. 27 de diciembre de 1990. Id SAIJ: FA90000451 
al Estado a restringir el ejercicio normal de algunos derechos patrimoniales tutelados por la Constitución Nacional.

Así, la Corte Suprema de Justicia brinda el marco para definir la emergencia, más allá del hecho de que la motive, autorizando la utilización de remedios excepcionales para poner fin a ella.

Del mismo modo, en la recepción de la emergencia el máximo tribunal de justicia reconoce la validez de la restricción del ejercicio normal de derechos tutelados en la Constitución.

Ahora bien, se observa la singular importancia del rol del intérprete, toda vez que será quien defina:

1) situación excepcional,

2) validez de los remedios extraordinarios, y

3) razonabilidad de la limitación de los derechos.

Vale la aclaración fundamental de que hablamos de restricción de derechos y no de su desconocimiento, ni tampoco de una habilitación para apartarse de la normativa vigente.

\section{MARCO JURÍDICO DE LA EMERGENCIA}

La Ley 27541, que declaró la emergencia sanitaria, señala en su parte pertinente:

Artículo $1^{\circ}$ - Declárase la emergencia pública en materia económica, financiera, fiscal, administrativa, previsional, tarifaria, energética, sanitaria y social, y deléganse en el Poder Ejecutivo nacional, las facultades comprendidas en la presente ley en los términos del artículo 76 de la Constitución Nacional, con arreglo a las bases de delegación establecidas en el artículo $2^{\circ}$, hasta el 31 de diciembre de 2020.
Artículo $2^{\circ}$ - Establécense las siguientes bases de delegación: (...) f) Procurar el suministro de medicamentos esenciales para tratamientos ambulatorios a pacientes en condiciones de alta vulnerabilidad social, el acceso a medicamentos e insumos esenciales para la prevención y el tratamiento de enfermedades infecciosas y crónicas no trasmisibles; atender al efectivo cumplimiento de la Ley 27491 de control de enfermedades prevenibles por vacunación y asegurar a los beneficiarios del Instituto Nacional de Servicios Sociales para Jubilados y Pensionados y del Sistema Nacional del Seguro de Salud, el acceso a las prestaciones médicas esenciales.

En definitiva, es la norma la que establece el marco y alcance de los decretos que se dicten en razón de la aludida emergencia.

En virtud de la delegación efectuada por la norma, se dictaron, entre otros, el Decreto 260/20 que amplía la emergencia pública en materia sanitaria establecida por Ley 27541, en virtud de la pandemia declarada por la Organización Mundial de la Salud (OMS) en relación a la COVID-19, por el plazo de UN (1) año a partir del 13 de marzo del año 2020 y sus sucesivas prórrogas, la última de ellas a través del Decreto $167 / 2021$ (que prorroga la emergencia sanitaria hasta el 31 de diciembre del año 2021); el Decreto 297/20, que dispuso «aislamiento social, preventivo y obligatorio» (ASPO) hasta el 31 de marzo incluido; y el Decreto 320/20 que dispuso la suspensión de desalojos, la prórroga de contratos de locaciones y el congelamiento de cánones locativos, hasta el día 30 de septiembre de 2020, de las viviendas que la misma norma estipula 3 .

Se dictó, asimismo, y siempre haciendo especial hincapié en aquellos decretos que se

3 El mencionado decreto ha sido prorrogado a enero de 2021. 
vinculan con el objeto del presente, el Decreto de Necesidad y Urgencia $457 / 20$ por el cual se modifica el presupuesto general de la administración, facultando al jefe del gabinete de ministros a realizar reestructuraciones presupuestarias, exceptuándolo de cumplir con lo dispuesto por el tercer párrafo del artículo 37 de la Ley 24156 de Administración Financiera y de los Sistemas de Control del Sector Público Nacional.

Es aquí donde el Congreso debe asumir su función de contralor toda vez que la modificación del presupuesto (aprobado por ley) no puede habilitar, aun en situaciones de emergencia, su desnaturalización vía reestructuraciones realizadas por el jefe de gabinete de ministros, eximiéndole del cumplimiento de las pautas (y límites) dispuestas por la misma Ley 24156.

\section{VALIDEZ CONSTITUCIONAL Y CONVENCIONAL DE LA EMERGENCIA}

La Corte Suprema de Justicia de la Nación en autos «Avico, Oscar A. c/ De la Pesa, Saúl»» ha señalado los requisitos para la validez de los institutos de emergencia:

Es necesario para ello: 1) que exista una situación de emergencia que imponga al Estado el deber de amparar los intereses vitales de la comunidad; 2 ) que la ley tenga como finalidad legítima, la de proteger los intereses generales de la sociedad y no a determinados individuos; 3) que la moratoria sea razonable, acordando un alivio justificado por las circunstancias; 4) que su duración sea temporal y limitada al plazo indispensable para que desaparezcan las causas que hicieron necesaria la moratoria.

\footnotetext{
${ }^{4}$ Fallos: 172:21. 7 de diciembre de 1934. Id SAIJ: FA34996938
}

En esta situación, la declaración de la Organización Mundial de la Salud y los informes de las víctimas fatales per se justifican la declaración de emergencia efectuada:

Es claro que la finalidad del aislamiento social como la afectación de los demás derechos es de carácter general y con la finalidad de asegurar el bien común.

Asimismo, la duración en el tiempo es razonable. En lo respectivo al aislamiento social, a la prórroga de contratos de locación y suspensión de los desalojos, estos deben ser acotados a la situación de gravedad sanitaria, en atención a los efectos de estas medidas sobre el sector privado.

El análisis de la razonabilidad también debe efectuarse sobre las medidas que se dicten en el marco de la emergencia aludida. En este sentido, el profesor Gordillo señala que:

La norma legal o reglamentaria (...) al igual que la decisión individual, actuación material u omisión de las administraciones públicas y del estado en general, será ilegítima, a pesar de no transgredir ninguna norma concreta y expresa, si es «irrazonable», lo que puede ocurrir, principalmente, cuando: a. No dé en el acto particular los fundamentos de hecho o de derecho que lo sustentan; b. No tenga en cuenta en las normas o en las decisiones concretas, los hechos que constan en el expediente, o públicos y notorios; o se fundamente en hechos o pruebas inexistentes; 0

c. No guarde una proporción adecuada entre los medios que emplea y el fin que la ley desea alcanzar, es decir, que se trate de una medida desproporcionada, excesiva en relación a lo que se quiere alcanzar (...) (GordiIlo, 2014, pp. 618 y 817). 
Es en este último aspecto en el que entendemos que debemos detenernos, al incorporar el criterio de proporcionalidad, cuya falta de resguardo torna irrazonable a la medida adoptada en el marco de la excepcionalidad.

Podemos definir al principio de proporcionalidad como a la relación debida y adecuada entre los medios utilizados y el fin buscado por la norma, que limita el poder del Estado a la hora del ejercicio del poder de policía.

La Comisión Interamericana de Derechos Humanos, en su Resolución 1/2020, específicamente en sus puntos 20 y 21 , señala:

20. Asegurar que toda restricción o limitación que se imponga a los derechos humanos con la finalidad de protección de la salud en el marco de la pandemia COVID-19 cumpla con los requisitos establecidos por el derecho internacional de los derechos humanos. En particular, dichas restricciones deben cumplir con el principio de legalidad, ser necesarias en una sociedad democrática $y$, por ende, resultar estrictamente proporcionales para atender la finalidad legítima de proteger la salud.

21. Asegurar que en caso de establecerse un estado de excepción: i) se justifique que existe una excepcionalidad de la situación de emergencia en cuanto a su gravedad, inminencia e intensidad que constituye una amenaza real a la independencia o seguridad del Estado; ii) la suspensión de algunos derechos y garantías sea únicamente por el tiempo estrictamente limitado a las exigencias de la situación; iii) las disposiciones que sean adoptadas resulten proporcionales, en particular, que la suspensión de derechos o garantías constituya el único medio para hacer frente a la situación, que no pueda ser enfrentada mediante el uso de las atribuciones ordinarias de las autoridades estatales, y que las medidas adoptadas no generen una mayor afectación al derecho que sea suspendido en comparación con el beneficio obtenido; y iv) las disposiciones adoptadas no sean incompatibles con las demás obligaciones que impone el derecho internacional, y no entrañen discriminación alguna fundada, en particular, con motivos de raza, color, sexo, idioma, religión u origen social.

La Comisión establece claramente cuáles son los estándares de validez convencional que debe respetar toda normativa que se dicte en el marco de la pandemia de la COVID-19. Este aporte, que entendemos fundamental atento a lo oportuno de su intervención, permite conocer el marco sobre el que se juzgará la convencionalidad o no de la normativa que se dicte en el marco de la emergencia sanitaria.

\section{EL ESTADO DE DERECHO}

Hoy la idea del Estado de derecho ha sido ampliada, avanzando sobre lo ya expuesto sobre el Estado constitucional y convencional de derecho.

El doctor Andrés Gil Domínguez señala que

El paradigma argentino vigente es el Estado constitucional y convencional de derecho, el cual escenifica como elemento central la Constitución y los instrumentos internacionales sobre derechos humanos que ostentan jerarquía constitucional en una relación de permanente retroalimentación donde se respeta la textualidad de cada fuente y la interpretación que cada órgano con competencia para ello realiza de las mismas.

Entonces, analicemos someramente qué establecen la Constitución y la Convención Americana de Derechos Humanos al respecto. 
La Constitución no solo garantiza derechos y garantías, sino que determina el carácter relativo de los derechos. Así, en su artículo 14 establece «conforme a las leyes que reglamenten su ejercicio».

En ese sentido, la Convención Americana de Derechos Humanos sostiene en su artículo 22 inciso 3 que el ejercicio de los derechos a circular y residir en un Estado consagrados en el artículo 22.1:

... no puede ser restringido sino en virtud de una ley, en la medida indispensable en una sociedad democrática, para prevenir infracciones penales o para proteger la seguridad nacional, la seguridad o el orden públicos, la moral o la salud públicas o los derechos y libertades de los demás.

Por su parte, la Comisión Interamericana de Derechos Humanos (CIDH) dicta la Resolución 1/2020 atenta a que la pandemia de la COVID-19

... puede afectar gravemente la plena vigencia de los derechos humanos de la población en virtud de los serios riesgos para la vida, salud e integridad personal que supone la COVID-19; así como sus impactos de inmediato, mediano y largo plazo sobre las sociedades en general, y sobre las personas y grupos en situación de especial vulnerabilidad (...). En cuanto a las medidas de contención con el fin de enfrentar y prevenir los efectos de la pandemia, la CIDH ha observado que se han suspendido y restringido algunos derechos, y en otros casos se han declarado "estados de emergencia», "estados de excepción», «estados de catástrofe por calamidad pública», o «emergencia sanitaria», a través de decretos presidenciales y normativa de diversa naturaleza jurídica con el fin de proteger la salud pública y evitar el incremento de contagios. Asimismo, se han establecido medidas de distinta naturaleza que restringen los derechos de la libertad de expresión, el derecho de acceso a la información pública, la libertad personal, la inviolabilidad del domicilio, el derecho a la propiedad privada; y se ha recurrido al uso de tecnología de vigilancia para rastrear la propagación del coronavirus, y al almacenamiento de datos de forma masiva.

La Comisión busca que en el marco de la emergencia sanitaria esta no opere como una excusa para el avasallamiento y la afectación de derechos fundamentales.

Las recomendaciones que vierte apuntan a una reglamentación de la emergencia y son una valiosísima pauta interpretativa a la hora de poder analizar la validez convencional de las medidas que se adopten en los diferentes Estados miembros.

El hecho de que esta resolución se dicte de manera simultánea con las medidas que se adoptan para paliar los efectos de la pandemia es muestra de que el control se puede realizar de manera eficaz pero también oportuna, esto es, simultáneamente con la gestión estatal.

\section{EL CONTROL DE LA EMERGENCIA}

Como se observa de lo hasta aquí presentado, estamos ante una situación de emergencia cuya validez constitucional y convencional se encuentra, a nuestro entender, debidamente acreditada.

Ahora bien, cabe preguntarnos si en este contexto es necesario o no el funcionamiento de los órganos de control, y de qué modo este debe ejercerse.

Es indudable que a la primera pregunta la respuesta es «sí», por todos los fundamentos 
que justifican la existencia del control y también porque resulta un reclamo genuino de la sociedad hacia el Estado y sus organismos de control.

Así surge de lo informado por el área de Participación Ciudadana de la Auditoría General de la Nación (AGN), que en su encuentro anual para presentar los resultados del Programa de Planificación Participativa recibió como una de las propuestas de las instituciones asistentes el realizar un control específico sobre las compras y contrataciones efectuadas en el marco de la pandemia por la COVID-19.

Podemos reconocer aquí un genuino interés de la sociedad en conocer la administración y, sobre todo, la ejecución de los fondos públicos utilizados para atender la pandemia.

En este punto es dable recordar que la Resolución 1/2020 de la Comisión Interamericana de Derechos Humanos señala que

Teniendo en cuenta que la democracia y el Estado de derecho son condiciones necesarias para lograr la vigencia y el respeto de los derechos humanos, y que la naturaleza jurídica de las limitaciones a dichos derechos puede tener impactos directos en los sistemas democráticos de los Estados, la Comisión reafirma el rol fundamental de la independencia y de la actuación de los poderes públicos y las instituciones de control, en particular de los poderes judiciales y legislativos, cuyo funcionamiento debe ser asegurado aun en contextos de pandemia.

La resolución citada evidencia la necesidad de que los países que forman parte de la Convención Americana de Derechos Humanos garanticen la vigencia de los derechos fundamentales incluso en épocas de pandemia, lo que puede significar una afectación de derechos pero no su supresión o desconocimiento.
La emergencia no implica impunidad, sino todo lo contrario; siendo una oportunidad para demostrar que aun en situaciones extremas el Estado puede responder de manera oportuna ante necesidades que ameritan respuestas inmediatas.

Por eso, y en respuesta al segundo interrogante planteado al inicio de este acápite, la participación e intervención de los organismos de control conlleva necesariamente un análisis particularizado que coadyuve al cumplimiento de la legalidad de los trámites y a la celeridad requerida.

La pandemia que atraviesa nuestras vidas ha requerido un grado de intervención estatal más activa. Es claro que esta mayor intervención no debería habilitar el incumplimiento de la normativa, es decir, no debería justificar mayor arbitrariedad por parte del Estado a la hora de contratar la adquisición de bienes y/o de servicios para hacer frente a los gastos para atender la pandemia, con la urgencia que se requiere sin que implique un apartamiento de la legislación vigente. Por eso, es necesario e imprescindible la actuación de los organismos políticos y técnicos de control.

Como hemos sostenido en otras oportunidades, la burocracia siempre importa un criterio de orden en la actuación de la Administración, que da una mayor previsibilidad en su accionar en aras del cumplimiento de la seguridad jurídica siempre anhelada. El control de esa burocracia en esta situación de emergencia debe estar aún más presente, ya que su rol de coadyuvante de la Administración se torna imprescindible.

Entender que el control solo debe sancionar importa una mirada sesgada y retrograda de una función que tiene como principal finalidad la protección de los intereses de la sociedad mediante la prevención de la corrupción administrativa. 
En situaciones como las que nos tocan vivir se torna imprescindible dar soluciones con la celeridad que se requiere, pero siempre dentro de la legalidad propia de la Administración.

Las distintas instancias de control deben estar, a nuestro entender, más presentes que nunca.

Así, propongo que analicemos estos aspectos desde dos ejes distintos: por un lado, un «control político»; por otro, un «control técnico».

El control político es el que se realiza entre los distintos poderes, esto es, el Legislativo principalmente sobre el Ejecutivo.

La emergencia declarada por la Ley 27541 establecía las bases de la delegación efectuada, ello importa un límite a la actividad normativa que va a poder desarrollar el Ejecutivo; límite que, a nuestro entender, resulta muy dificultoso determinar atento a la amplitud de las materias objeto de la emergencia.

Más aun, la emergencia declarada, como se ha dicho, es anterior a la declaración de pandemia por parte de la Organización Mundial de la Salud de la COVID 19, que sirve de fundamento a todos los decretos dictados con posterioridad.

La Ley 26122, que determina la intervención del Congreso en los decretos que dicta el Poder Ejecutivo, establece límites muy precisos en la delegación legislativa, por ejemplo en sus artículos 11 y 12, el primero referido a los alcances de los decretos delegados y el segundo respecto del procedimiento para su dictado.

Es en el artículo 13 donde encontramos el control político sobre estos:

La Comisión Bicameral Permanente debe expedirse acerca de la validez o invalidez del decreto y elevar el dictamen al plenario de cada Cámara para su expreso tratamiento. El dictamen debe pronunciarse expresamente sobre la procedencia formal y la adecuación del decreto a la materia y a las bases de la delegación, y al plazo fijado para su ejercicio. Para emitir dictamen, la Comisión Bicameral Permanente puede consultar a las comisiones permanentes competentes en función de la materia.

Esta herramienta de control resulta imprescindible para determinar la validez constitucional de los decretos dictados en el marco de la pandemia, a la hora de seguir los procedimientos establecidos para su ratificación legislativa.

El control político por parte del Poder Legislativo también se puede ejercer a través del pedido de informes a los funcionarios del Poder Ejecutivo, y hasta en su interpelación cuando se lo considere necesario y oportuno como un modo de exigir la rendición de cuentas de su accionar a la sociedad.

Es tan importante que incluso en la emergencia se asegure el funcionamiento de los distintos poderes del Estado que, adecuándose al estatus sanitario de nuestro país, ambas cámaras del Congreso habilitaron su funcionamiento remoto.

Esto permitió su funcionamiento y también que, a través del jefe de gabinete de ministros, en virtud de lo señalado por el artículo 101 de la Constitución Nacional, se haya informado mensual y alternadamente a cada Cámara sobre la marcha del Gobierno, donde el eje central ha sido lo atinente a la atención de los gastos derivados de la pandemia. Esto también es un ejercicio efectivo de las funciones de control.

El seguimiento y la publicidad de la ejecución presupuestaria de los distintos programas y jurisdicciones específicamente afectados para atender a los efectos de la pandemia constituyen otra herramienta eficaz de control. Así, la publicación de los fondos ejecutados, su 
destino y su posterior rendición a los organismos de control es una manera de hacer efectivo y eficaz al control: transparencia e información, principios que se vinculan con el mismo sistema republicano de gobierno.

En lo referente al «control técnico» lo señalamos como aquel que realizan los organismos de control tanto internos como externos de la Administración.

El control que comienza dentro de la misma organización a partir de sus órganos técnicos especializados, los abogados en sus dictámenes, los profesionales o idóneos a la hora de la afectación de los fondos y su proceso de contratación, lo que denominamos control interno, adquiere una importancia relevante.

Núñez lo define como aquel que representa

... aquella actividad de supervisión destinada a preservar la regularidad y eficiencia de la gestión de gobierno. Es un autocontrol que los mismos órganos ejecutivos practican para asegurarse el logro de los objetivos propuestos en los distintos planes de realización de obras y servicios, o regulación de la comunidad sujeta a su gobierno, como manera de preservar la legalidad de lo actuado (regularidad de gestión), como asimismo el cumplimiento de las acciones en cantidad, tiempo y forma (oportunidad y conveniencia) (Núñez, 2006, p. 56).

La importancia del control interno radica no solo en el carácter preventivo y correctivo, según corresponda, de su intervención, sino que de algún modo replica los criterios fijados por los organismos de control externo para unificar criterios o interpretación de normativa, como así también de procedimientos.
Para poder observar cómo se ha previsto en otros países realizar el control sobre los fondos asignados para atender a los gastos de la pandemia podemos citar el ejemplo de Perú, donde la Contraloría General de ese país publica el seguimiento de la ejecución presupuestaria sobre los fondos asignados para atender a la pandemia de la COVID-19, realizando un control simultáneo de las compras y contrataciones realizadas 5 .

En nuestro país el control interno es el que realiza la Sindicatura General de la Nación, en el ámbito de las competencias asignadas por la Ley 24156 (ley de administración financiera).

El control externo es el que realiza la Auditoría General de la Nación, conforme lo establece el artículo 85 de nuestra Constitución Nacional.

Las auditorías que realiza este organismo son las que surgen del Plan Operativo Anual aprobado para el organismo por la Comisión Mixta Revisora de Cuentas.

Al no estar incluido el control sobre los gastos e inversiones derivados en la pandemia en el Plan Operativo Anual 2020, estos no fueron controlados por la Auditoría General de la $\mathrm{Na}$ ción durante el año 2020.

Esto último demuestra la necesidad de otorgarles a los organismos de control la autonomía suficiente para que puedan desarrollar sus tareas con la dinámica propia que el devenir de la gestión gubernamental desarrolla. Esto no significa que estos gastos queden sin control, sino que ese control no pueda realizarse simultáneamente con la gestión.

El control, para ser eficaz debe ser oportuno; realizarlo de manera posterior solo sirve para determinar responsabilidades (administrativas, disciplinarias y hasta penales) pero en un contexto de pandemia entendemos que,

\footnotetext{
5 El informe se puede consultar en la página web: https://emergenciasanitaria.contraloria.gob.pe/
} 
como se habilitaron vías excepcionales de contratación, debió haberse previsto un mecanismo de control simultáneo.

No era necesario crear ni una comisión especial ni un nuevo organismo de control, solo habría bastado con habilitar la intervención de la Auditoría General de la Nación, que por su carácter de órgano constitucional de control y por contar con el personal especializado, hubiera podido ejercer una misión tan importante en salvaguarda del interés general.

Si la pandemia permitió reivindicar el rol activo del Estado también lo podría haber hecho con los organismos de control.

Era una oportunidad, primero, para visibilizar los órganos de control ante la sociedad toda; y segundo, era una manera de asegurar la legalidad de las contrataciones realizadas.

El control posee distintas instancias de intervención, puede ser previo (antes de que el acto se dicte), preventivo (una vez que el acto se dicta pero antes de que surjan sus efectos), por auditorías (más allá del tipo de auditoria implica una verificación casi simultánea con la ejecución), y posterior (a través de la rendición documentada de los gastos que se realicen).

Tanto el control previo como el control por auditorías han demostrado ser herramientas fundamentales de prevención de la corrupción. Al eliminarse en nuestro país el Tribunal de Cuentas de la Nación, que era quien ejercía dicho control, debió preverse la presencia de este control a través de la Auditoría General de la Nación.

La realidad provincial es distinta, toda vez que en la mayoría de las provincias (con excepción de Salta y de la Ciudad Autónoma de Buenos Aires, que poseen auditorías generales) el control lo realizan los tribunales de cuentas provinciales.

La crítica más frecuente hacia los organismos de contralor es que su intervención, so- bre todo en lo que respecta al control previo, produce una innecesaria dilación de los plazos, máxime en una situación de emergencia.

Sin embargo esta crítica no tiene, a nuestro entender, fundamento que justifique promover la exclusión de este tipo de control.

El carácter perentorio de los plazos, previsto por la normativa para sus intervenciones, coadyuva para que no pueda señalarse como un obstáculo para el trámite de las actuaciones.

Es el desafío de los organismos intervinientes demostrar que en una situación de emergencia las contrataciones para la adquisición de equipamiento e infraestructura, como también de insumos para atenderla, pueden realizarse de manera expedita y sin afectar su legalidad.

\section{REFLEXIONES FINALES}

El presente trabajo busca presentar el control como una herramienta imprescindible de buena gestión y transparencia, máxime en una situación de emergencia donde están en juego derechos humanos fundamentales como el de la vida y la salud de la población.

Muchas veces hemos escuchado, por parte de quienes tienen la responsabilidad de gestionar, que el control (en sus distintas instancias) resulta un entorpecedor de la gestión, atento a que retarda la tramitación de las actuaciones administrativas. Ello no es cierto, ya que el control, como hemos presentado, resulta una herramienta imprescindible de buena gestión que garantiza la vigencia del mismo sistema republicano de gobierno.

Por ello, tanto los poderes del Estado como los órganos de contralor deben dar respuestas eficaces y oportunas sin que ello implique una dilación innecesaria en los trámites de contratación del Estado.

Que quede claro que no nos referimos a co-administrar o realizar un control de gestión, 
sino a acompañar la función de control en lo que respecta a la legalidad de la actuación.

El control no es un obstáculo sino que es la oportunidad de legitimar decisiones que no por inmediatas deben justificar irregularidad alguna.

En la medida en que las decisiones adoptadas en el marco de una emergencia tengan un marco legal y prevean la intervención de todas las instancias de control, dichas decisiones harán efectiva la garantía de la seguridad jurídica tanto para el funcionario que las adopte como para el tercero que contrate con la Administración.

El funcionamiento de todos los poderes del Estado opera también como un modo de hacer efectivo el control en épocas de pandemia.

La emergencia constituye una oportunidad también para poder legitimar los distintos poderes del Estado, pero también el funcionamiento de los órganos de control.

El Estado nacional, al haber centralizado la compra de insumos (por ejemplo, las vacunas) e insumos (por ejemplo, los respiradores), derivándolos a las distintas jurisdicciones, debería haber instrumentado la intervención de los organismos de control externo aun en un contexto de pandemia.

Poder y control constituyen cara y contracara de la misma moneda, pese a que en esta instancia haya sido mucho más visible el poder de la Administración.

Celeridad para la gestión y oportunidad para el control, y cuanto más inmediato este sobre la ejecución presupuestaria más eficaz será.

Es en este sentido que entendemos que a nivel nacional debería haberse generado alguna instancia de control externo lo más inmediato posible sobre la ejecución de los fondos destinados para afrontar los gastos que la pandemia originó.
No significa que están exentos del control, sino que los gastos derivados para la atención de la COVID-19 van a ser objeto de auditorías por lo menos un año después de haber sido ejecutados, con lo cual el carácter oportuno del control (que hace a su eficacia) no se pudo ejercer.

Para concluir, podemos señalar que la emergencia también constituye una oportunidad de revisar procedimientos tanto de gestión como de control, para que podamos observar en la pandemia la posibilidad de avanzar hacia un mejor funcionamiento de nuestras instituciones que le otorgue a nuestras instituciones gubernamentales la siempre tan anhelada legitimidad ciudadana.

\section{REFERENCIAS BIBLIOGRÁFICAS}

Este año la AGN no controlará los gastos por la pandemia (2020). El auditor info. Recuperado de: https://elauditor.info/actualidad/esteano-la-agn-no-controlara-los-gastos-por-lapandemia_a5f0fb79e6b53fc3c338e3195

Gil Domínguez, A. (2014). El Estado constitucional y convencional de derecho argentino y sus proyecciones. DPI cuántico. Derecho integral. Recuperado de: http:// www.dpicuantico.com/wp-content/ uploads/2014/05/doctrina19.5.141.pdf. [Consulta: 30 de marzo de 2020]

Gordillo, A. (2014). Tratado de derecho administrativo. Tomo 9, separata. Buenos Aires: Fundación de Derecho Administrativo.

Núñez, J. A. (2006). Manual de auditoría gubernamental. Buenos Aires: Ediciones RAP.

¿Qué temas propuso auditar la sociedad civil? (2020). El auditor info. Recuperado de: https://elauditor.info/actualidad/-quetemas-propuso-auditar-la-sociedad-civil-_ a5fd21d7046a50103c357ab62 [Consulta: 14 de diciembre de 2020]. 